\title{
Nonsuicidal self-injury and suicide attempts in Iraq/Afghanistan war veterans
}

Nathan A. Kimbrel, ${ }^{\text {a, b, c* }}$ Bryann B. DeBeer, ${ }^{\text {d, e, f }}$ Eric C. Meyer, ${ }^{\text {d e, f }}$

Suzy B. Gulliver, ${ }^{\mathrm{g}} \&$ Sandra B. Morissette ${ }^{\mathrm{h}}$

${ }^{\text {a }}$ Durham Veterans Affairs Medical Center, Durham, NC, USA

${ }^{\mathrm{b}}$ VA Mid-Atlantic Mental Illness Research, Education, and Clinical Center; Durham, NC, USA

${ }^{\mathrm{c}}$ Duke University Medical Center, Durham, NC, USA

${ }^{\mathrm{d}}$ VISN 17 Center of Excellence for Research on Returning War Veterans, Waco, Texas, USA

${ }^{\mathrm{e}}$ Central Texas Veterans Health Care System, Temple, Texas, USA

${ }^{\mathrm{f}}$ Texas A\&M University Health Science Center, College Station, Texas, USA

${ }^{\mathrm{g}}$ Warriors Research Institute, Baylor, Scott \& White Healthcare System, Waco, TX, USA

${ }^{\mathrm{h}}$ The University of Texas at San Antonio, San Antonio, TX, USA

*Correspondence concerning this article should be sent to: Dr. Nathan A. Kimbrel, Durham

Veterans Affairs Medical Center, 508 Fulton Street, Durham, NC, 27705. Phone: (919)

286-0411, ext. 6759. Email: Nathan.Kimbrel@va.gov. 


\begin{abstract}
The present study examined the association between history of nonsuicidal self-injury (NSSI) and history of suicide attempts (SA) among 292 Iraq/Afghanistan veterans, half of whom carried a lifetime diagnosis of posttraumatic stress disorder (PTSD). Consistent with hypotheses, veterans who reported a history of NSSI were significantly more likely to report a history of SA than veterans without a history of NSSI. In addition, logistic regression demonstrated that NSSI remained a significant predictor of SA even after a wide range of covariates (i.e., combat exposure, traumatic brain injury, PTSD depression, alcohol dependence) were considered. Taken together, these findings suggest that clinicians working with veterans should include NSSI history as part of their standard risk assessment battery.
\end{abstract}

Keywords: Nonsuicidal self-injury; suicide; suicide attempts; suicidal ideation; PTSD; depression; veterans

\title{
1. Introduction
}

Nonsuicidal self-injury (NSSI) refers to the act of intentionally destroying one's own body tissue without conscious suicidal intent (Chapman et al., 2006; Suyemoto, 1998). It is estimated that approximately 4-6\% of adults in the general population engage in NSSI at some point during their lifetime (Briere and Gil, 1998; Klonsky, 2011). However, lack of a precise nomenclature for NSSI often leads to differing rates across populations (Crosby et al., 2011; Silverman et al., 2007). Common NSSI methods include cutting (35\%), burning (31\%), hitting (31\%), and biting (31\%; Klonsky, 2011), although approximately half of individuals who engage in NSSI use multiple methods (Klonsky, 2011). Notably, population-based studies indicate that NSSI is not related to gender, ethnicity, income, or education (Briere and Gil, 1998; Klonsky, 
2011). These findings challenge the long-held belief that NSSI is more common in women (e.g., Miller, 1994), and indicate that this issue exists in a multitude of populations. NSSI is, however, significantly more common among individuals with a history of mental health treatment (Bentley et al., 2015; Briere and Gil, 1998; Klonsky, 2011). For instance, Briere and Gil (1998) reported that $21 \%$ of a psychiatric patient sample ( $73 \%$ of whom carried a diagnosis of PTSD) reported engaging in NSSI during the previous 6 months.

\subsection{Nonsuicidal self-injury in veterans}

Rates of NSSI in non-treatment seeking veteran samples range from $2.8 \%$-14\% (Bryan and Bryan, 2014; Kimbrel et al., 2015; Pinder et al., 2011; Swanell et al., 2014). However, rates of NSSI in veterans seeking treatment for PTSD appear to be markedly higher. For example, Sacks and colleagues (2008) examined rates of NSSI among a sample of primarily Vietnam-era veterans seeking treatment for PTSD and found that $66 \%$ reported a lifetime history of NSSI, whereas 55\% reported engaging in NSSI during the past two weeks. More recently, Kimbrel and colleagues (2014a) examined the prevalence of NSSI among a sample of male Iraq/Afghanistanera veterans seeking treatment for PTSD and found that 57\% reported lifetime NSSI, whereas $45 \%$ reported NSSI during the previous two weeks. These findings are consistent with findings from the civilian literature demonstrating that PTSD is one of the psychiatric disorders that is most strongly associated with NSSI (e.g., Bentley et al., 2015).

\subsection{Nonsuicidal self-injury and suicidal behavior}

While inherent in the definition of NSSI is a lack of suicidal intent, a strong body of research among civilian samples indicates that NSSI is consistently associated with increased 
risk for engaging in suicidal behavior (e.g., Asarnow et al., 2011; Hamza et al., 2012; Klonsky et al., 2013). For example, Klonsky and colleagues (2013) recently demonstrated that NSSI was more strongly associated with history of suicide attempts (SA) than depression, anxiety, and borderline personality disorder (BPD) across multiple civilian samples. These and other similar findings (e.g., Asarnow et al., 2011; Hamza et al., 2012) are consistent with Joiner's (2005) proposal that NSSI increases risk for suicidal behavior by increasing individuals' acquired capability for suicide. Specifically, the repetition of bodily harm is proposed to enhance individuals' tolerance of physiological pain and to diminish their fear of death and/or bodily harm, which, in turn, is proposed to increase their capability to engage in suicidal behavior (Joiner, 2005; Nock et al., 2006).

To date, there has been only limited research aimed at examining the association between NSSI and suicidal behavior in veterans. Specifically, Kimbrel and colleagues (2014a; 2015a) have shown that NSSI is associated with increased risk for suicidal ideation in two cohorts of Iraq/Afghanistan-era veterans, whereas Bryan and colleagues (2015) recently demonstrated that NSSI was prospectively associated with subsequent SA $(H R=2.25, p<0.05)$ among 152 activeduty soldiers with a recent history of suicidal ideation or SA.

\subsection{Study objective}

Given the increasing evidence that veterans with PTSD and other mental health disorders are at elevated risk for suicidal behavior and death by suicide (e.g., Bullman and Kang, 1994; DeBeer et al., 2014; Kang and Bullman, 2008; U.S. Department of Veterans Affairs, 2013; Kaplan, Huguet, McFarland, and Newsom, 2007; Kimbrel et al., 2014b), there is a significant need for additional studies aimed at elucidating the association between NSSI and suicide 
attempts in veterans, as this important association has only been examined in one prior study with an active duty military sample (Bryan et al., 2015). Accordingly, the aim of the present research was to examine the association between history of NSSI and history of SA in a crosssectional sample of 292 Iraq/Afghanistan-era veterans. Consistent with prior research in this area (e.g., Bryan et al., 2015; Klonsky et al., 2013), we hypothesized that NSSI would be associated with risk for SA at the bivariate level. We further expected that the hypothesized association between NSSI and SA would remain significant after a wide range of potential confounding variables were considered. Specifically, since gender, age, race, sexual orientation, combat exposure, PTSD, major depressive disorder (MDD), alcohol dependence, and traumatic brain injury (TBI) have all been previously associated with suicidal behavior (Kessler et al., 1999; Nock and Kessler, 2006; Nock et al., 2010; Remafedi et al., 1998; Bryan et al., 2013; Martin et al., 2012; Blosnich et al., 2012), we included these covariates in the logistic regression model to determine if NSSI would be uniquely associated with SA above and beyond the effects of these established predictors.

\section{Method}

\subsection{Participants}

A total of 345 Iraq/Afghanistan-era veterans were recruited from the Central Texas region of the United States to participate in the parent study. To be eligible, participants had to be U.S. veterans who had deployed in support of the conflicts in Iraq and Afghanistan following September $11^{\text {th }}, 2001$. Participants could not meet criteria for either bipolar disorder or a psychotic disorder. Participants were also required to be stable on medications and therapy at the 
baseline assessment to control for recent symptom fluctuations. In addition, participants could not be in acute suicidal crisis necessitating immediate intervention, nor could they be planning on moving out of the catchment area in the immediate future, as the primary goal of the parent study was to study long-term functional recovery among Iraq/Afghanistan-era veterans with more common mental health problems, particularly PTSD and depression. Accordingly, veterans with PTSD and depression (but without a diagnosis of bipolar disorder or psychosis) who were enrolled in the VA healthcare system were oversampled through targeted advertisements, mailings, and referrals from clinical providers. Women were also oversampled.

Using these criteria, a total of 309 participants were deemed eligible for the parent study (21 were ruled out due to bipolar/psychosis, 13 were unable to complete all of the assessment procedures, 1 was not an Iraq/Afghanistan-era veteran, and 1 was planning on relocating in the near future). In addition, because data concerning lifetime history of NSSI and SA were not collected from 17 of the 309 Iraq/Afghanistan-era veterans enrolled in the parent study, the current analyses were limited to the 292 eligible Iraq/Afghanistan-era veterans who had complete NSSI and SA history data available for analysis from the baseline assessment.

Approximately one-third (32.5\%) of the sample was female. Approximately one-fourth of the sample was Latino (19.5\%). The majority of participants were White/Caucasian (56.8\%); however, African Americans (33.2\%) and American Indians/Alaska Natives (5.8\%) were also well represented. With respect to sexual orientation, $95.8 \%$ of the sample identified as heterosexual, $1.4 \%$ as homosexual, $0.7 \%$ as bisexual, $0.3 \%$ were unsure, and $0.3 \%$ classified themselves as "other" (categories are not mutually exclusive). In addition, approximately 3\% ( $n$ $=9$ ) of the sample chose to not answer the question concerning their sexual orientation. On average, participants had $14.2(S D=2.1)$ years of education. The majority $(86.5 \%)$ of 
participants had served in the served in the Army; $6.3 \%$ had served in the Air Force, 5.1\% in the Marine Corps, $2.8 \%$ in the Navy, and $8.7 \%$ in the National Guard (categories were not mutually exclusive).

\subsection{Procedures}

All procedures were approved by the Institutional Review Board prior to data collection. Following informed consent, participants completed the battery of clinical interviews and selfreport measures described below. All interviews were conducted by clinical psychologists and masters-level clinicians who were required to complete extensive training procedures prior to being allowed to conduct interviews independently. In addition, all clinical interviews were reviewed during diagnostic review groups that were led by experienced clinical psychologists. Diagnostic consensus was reached on all cases.

\subsection{Measures}

The Columbia Suicide Severity Rating Scale (CSSRS; Posner et al., 2011) was used to assess lifetime history of NSSI and SA. Consistent with the Self-Directed Violence Classification System used in the VA healthcare system, NSSI was defined as non-suicidal selfdirected violence, and SA were defined as suicidal self-directed violence (Crosby et al., 2011). Note that the current analyses were restricted to actual SA only. Thus, interrupted and aborted SA were not included in the SA variable used in the present analyses. The Clinician Administered PTSD Scale for DSM-IV (CAPS-IV; Blake et al., 1995) was used to diagnose lifetime PTSD in relation to the most severe event identified during participants' military service as part of the Iraq and Afghanistan conflicts. The Structured Clinical Interview for DSM-IV 
(SCID-IV; First et al., 1994) was used to diagnose lifetime MDD and alcohol dependence. A clinician administered TBI interview (Vasterling, 2008; Morissette et al., 2011) based on American Congress of Rehabilitation Medicine guidelines (Gerberding and Binder, 2003) was used to assess TBI. The Critical Warzone Experiences Scale (CWE; Kimbrel et al., 2014c) was used to assess combat exposure. Note that the PTSD, depression, alcohol dependence, TBI, NSSI, and SA variables used in the present analyses were all lifetime history variables.

\subsection{Data analytic plan}

Descriptive statistics were calculated for the clinical measures. Chi-square tests and ttests were used to examine bivariate associations between SA and predictors (Table 1). Hierarchical logistic regression was used to determine if NSSI would remain a significant predictor of SA after accounting for the potential influence of demographic variables [gender

$($ female $=0 ;$ male $=1)$, age, race $($ non-white $=0 ;$ white $=1)$, sexual orientation $($ heterosexual $=$ 0; non-heterosexual = 1)], combat exposure, TBI, and psychopathology (PTSD, MDD, and alcohol dependence). Lifetime history of SA served as the dependent variable. Gender, age, race, sexual orientation, and combat exposure were entered in step 1. TBI, PTSD, MDD, and alcohol dependence were entered in step 2. NSSI was entered in step 3.

\section{Results}

\subsection{Descriptive statistics}

Approximately $10 \%(\mathrm{n}=30)$ of the sample had a history of SA, 3\% $(\mathrm{n}=10)$ had a history of multiple SA, and 6\% $(n=18)$ had a history of NSSI. In addition, $50 \%(\mathrm{n}=145)$ of the 
sample met criteria for lifetime PTSD, 46\% $(\mathrm{n}=133)$ for lifetime depression, and $31 \%(\mathrm{n}=91)$ for lifetime alcohol dependence. In addition, 69\% $(n=200)$ of the sample had experienced one or more lifetime TBIs, and $64 \%(n=187)$ reported one or more combat experiences on the CWE.

\subsection{Bivariate analyses}

Bivariate associations between suicide attempts and predictors are provided in Table 1. Consistent with the main hypothesis, chi-square tests revealed that NSSI was strongly associated with history of SA, $\chi^{2}(1)=53.779, p<0.001$, including history of multiple SA, $\chi^{2}(1)=34.398, p$ $<0.001$, at the bivariate level. As can be seen in Figure 1, 61.1\% of veterans with a history of NSSI had attempted suicide at least once over the course of their lifetime. In addition, $27.8 \%$ of veterans with a history of NSSI had made multiple SA.

\subsection{Logistic regression model}

As can be seen in Table 2, gender, $O R=0.224, p=0.003$, age, $O R=0.910, p=0.001$, and combat exposure, $O R=1.996, p=0.005$, were significant predictors of SA in step 1 of the logistic regression model. Thus, being female, being younger, and having experienced higher levels of combat exposure were each associated with increased risk for SA. After adding TBI, PTSD, MDD, and alcohol dependence to the model in step 2, gender, $O R=0.240, p=0.015$, age, $O R=0.919, p=0.003$, and combat exposure, $O R=1.907, p=0.027$, continued to have significant effects on SA. Interestingly, $\mathrm{MDD}, O R=3.150, p=0.032$, was the only psychopathology variable significantly associated with SA in step 2. Finally, as hypothesized, NSSI was found to be strongly associated with SA in step 3 of the model, $O R=28.494, p<$ 
0.001. Notably, MDD no longer had a statistically significant effect on $\mathrm{SA}, O R=2.150, p=$ 0.183, once NSSI was added to the model in step 3, whereas gender, $O R=0.131, p=0.004$, age, $O R=0.926, p=0.009$, and combat exposure, $O R=3.204, p=0.002$, remained significant. The final model accounted for approximately $43 \%$ of the variance in SA (Nagelkerke $\left.R^{2}=0.429\right)$ and correctly classified $90.1 \%$ of participants.

\section{Discussion}

Consistent with the Interpersonal-Psychological Theory of Suicide (Joiner, 2005) and prior research examining the association between NSSI and SA (e.g., Bryan et al., 2015; Klonsky et al. 2013), the present study found that NSSI was associated with significantly increased risk for SA among Iraq/Afghanistan-era veterans at the bivariate level. Furthermore, the present study adds to the existing literature by demonstrating that NSSI remained predictive of SA, even after a wide range of covariates (e.g., demographic variables, combat exposure, TBI, psychiatric diagnoses) were considered. The latter finding is consistent with findings among civilian samples demonstrating that NSSI remained predictive of SA after accounting for anxiety and depression (Klonsky et al., 2013). The present findings, while cross-sectional in nature, are also consistent with Bryan and colleagues' (2015) recent finding associating NSSI with SA over time in activeduty military personnel.

Interestingly, the only other variables associated with risk for SA in the final step of the model were combat exposure, gender, and age. While each of these variables has been associated with suicidal behavior in previous research, it is surprising that none of the other established predictors of suicidal behavior examined (i.e., PTSD, MDD, alcohol dependence, TBI) were statistically significant predictors in the final step of the model. While it is possible that the 
categorical manner in which MDD, PTSD, alcohol dependence, and TBI were assessed may have reduced their predictive power, it is noteworthy that NSSI was also assessed categorically (i.e., present/absent), but still demonstrated a robust association with SA, even after all other variables had been included in the model.

It is also noteworthy that MDD had a statistically significant effect on SA until NSSI was entered into the model. Thus, it is likely that much of the variance that is shared between MDD and SA may also be shared with NSSI. Given that the rate of lifetime NSSI (6.2\%) was much lower than the rate of lifetime MDD (45.5\%) in the present study, it is likely that the substantially larger effect of NSSI on SA was due in large part to its specificity in relation to SA. That is, it is likely that NSSI, due to its much lower base rate in the present study, produced far fewer false positives than MDD. Such an interpretation is consistent with other recent findings in the field indicating that NSSI is a robust predictor of SA, outperforming depression and anxiety (e.g., Klonsky et al., 2013), and, in some cases, even outperforming history of SA as a predictor of future SA (e.g., Bryan et al., 2015).

Unfortunately, this important clinical issue has been largely overlooked among veteran populations to date. Thus, additional research aimed at further elucidating the association between NSSI and SA among veterans is needed, particularly given recent findings suggesting that NSSI is highly prevalent among both Vietnam- and Iraq/Afghanistan-era veterans seeking treatment for PTSD (Kimbrel et al., 2014a; Sacks et al., 2014).

A closely related point concerns the magnitude of the association between combat exposure $(O R=3.204, p<0.01)$ and SA observed in the present study, especially given the fact that combat exposure was assessed continuously. While there is clearly a strong theoretical rationale for why one might expect to find a strong relationship between combat and suicide 
attempts (i.e., because it increases acquired capability for suicide; Joiner, 2005; Selby et al., 2010), several prior studies have failed to find the expected association between combat and suicide risk (e.g., Bryan et al., 2013). Prior research has, however, found a fairly consistent association between combat exposure and acquired capability for suicide (e.g., Bryan and Cukrowicz, 2011; Bryan et al., 2013).

One potential explanation for the findings regarding combat and SA in the present study relates to the measure of combat exposure used. Specifically, to our knowledge, the current study is the first to examine the relationship between the CWE and suicidal behavior. This is particularly notable because the CWE was explicitly designed to assess warzone experiences that are strongly associated with the development of PTSD, depression, and anxiety in Iraq/Afghanistan-era veterans (Kimbrel et al., 2014). In addition, recent research has shown that this measure is also associated with a broad array of other types of psychiatric symptoms in veterans, including symptoms of panic disorder, obsessive-compulsive disorder, somatization, bulimia, and psychosis (Kimbrel et al., 2015b). It is possible that the unique set of warzone experiences assessed by the CWE may be more strongly associated with suicidal behavior than other warzone experiences. Preliminary support for this idea comes from the fact that, among other items, the CWE assesses the number of times that veterans were directly responsible for killing an enemy combatant during their deployment, as killing in combat has previously been found to be a unique predictor of suicidal ideation (Maguen et al., 2012). Clearly, additional research aimed at better understanding the relationship between different types of warzone experiences and suicidal behavior is needed. 


\subsection{Limitations}

The findings from the present study should be considered within the context of several limitations. First, the present study was cross-sectional and relied on retrospective assessment of NSSI and SA. Thus, causality cannot be inferred from the present findings. Moreover, while a high quality clinical interview (Posner et al., 2011) was used to assess both NSSI and SA, additional information regarding the onset and timing of NSSI and SA in relation to military service and deployments would have been useful in helping to disentangle these associations. A second limitation concerns the sample composition. Specifically, because veterans with PTSD and depression were oversampled in the present study, whereas veterans with a diagnosis of bipolar disorder or psychosis were excluded, the sample should not be considered representative of Iraq/Afghanistan-era veterans in general. Additional epidemiological work is needed to determine the true prevalence of NSSI among veterans. A final limitation concerns our limited diagnostic battery, which precluded us from assessing borderline personality disorder (BPD), a potentially important covariate that has been strongly associated with both NSSI and suicidal behavior in civilian samples (Klonsky et al., 2013; Linehan, 1993).

\subsection{Clinical implications}

Despite the limitations noted above, the present research, in conjunction with other recent studies in this area (e.g., Bryan et al., 2015; Kimbrel et al., 2014a, 2015a), has important implications for clinicians who routinely work with veterans of the conflicts in Iraq and Afghanistan. First, clinicians should be aware that NSSI does not appear to be limited by gender, ethnicity, income, education, or veteran status (Briere and Gil, 1998; Klonsky, 2011; Kimbrel et al., 2014a; Sacks et al., 2008). Thus, male veterans are as likely to engage in NSSI as female 
veterans or female civilians (e.g., Kimbrel et al., 2014a; Sacks et al., 2008). This is especially true for male veterans seeking treatment for PTSD (Kimbrel et al., 2014a; Sacks et al., 2008). Second, as demonstrated in the present study, clinicians should be aware that NSSI is one of the most robust predictors of SA identified to date (e.g., Asarnow et al., 2011; Bryan et al., 2015; Klonsky et al., 2013). As such, routine assessment of NSSI among veterans of the conflicts in Iraq and Afghanistan is advised, especially among Iraq/Afghanistan veterans who present with mental health concerns, such as PTSD. Third, while no research to date has attempted to treat NSSI among veterans, a growing body of research among civilians suggests that treatments that target emotion regulation skills (e.g., Linehan, 1993; Gratz et al., 2014) are likely to prove useful to these clients; however, additional work aimed at treating NSSI in veterans is still needed at the present time.

\subsection{Conclusion}

The findings from the present research suggest that NSSI is strongly associated with history of SA among Iraq/Afghanistan veterans, even after accounting for a number of important covariates, such as combat exposure, TBI, and psychopathology. More research on this important topic is needed. Prospective studies in high-risk samples of Iraq/Afghanistan veterans would be especially useful, as well as research aimed at determining if the findings from the present study extend to veterans of other eras. In the interim, the present findings clearly suggest that clinicians who routinely work with Iraq/Afghanistan veterans should make NSSI history a part of their standard risk assessment battery. 


\section{Acknowledgements}

This work was supported by a Merit Award (I01RX000304) to Dr. Morissette from the Rehabilitation Research and Development Service of the Department of Veterans Affairs (VA) Office of Research and Development (ORD), the VA VISN 17 Center of Excellence for Research on Returning War Veterans, the Central Texas Veterans Health Care System, the VA Mid-Atlantic Mental Illness Research, Education, and Clinical Center, and the Research \& Development and Mental Health Services of the Durham VA Medical Center. Dr. Kimbrel was supported by a Career Development Award (IK2 CX000525) from the Clinical Science Research and Development Service of the VA ORD. The views expressed in this article are those of the authors and do not necessarily reflect the position or policy of the Department of Veterans Affairs or the United States government. 


\section{References}

Asarnow J. R., Porta, G., Spirito, A., Emslie, G., Clarke, G., Wagner, K.D., et al., 2011. Suicide attempts and nonsuicidal self-injury in the treatment of resistant depression in adolescents: Findings from the TORDIA study. J Am Acad Child Adolesc Psychiatry. $50,772-781$.

Bentley, K.H., Cassiello-Robbins, C.F., Vittorio, L., Sauer-Zavala, S., Barlow, D.H. 2015. The association between nonsuicidal self-injury and the emotional disorders: A metaanalytic review. Clin Psychol Rev. 37, 72-88.

Blake, D., Weathers, F.W., Nagy, L.M., Kaloupek, D.G., Gusman, F.D., Charney, D.S. et al., 1995. The development of a Clinician-Administered PTSD Scale. J Trauma Stress. 8, 75-90.

Blosnich, J.R., Bossarte, R.M., Silenzio, V.M. 2012. Suicidal ideation among sexual minority veterans: Results from the 2005-2010 Massachusetts Behavioral Risk Factor Surveillance Survey. Am J Public Health. 102, S44-S47.

Briere, J., Gil, E., 1998. Self-mutilation in clinical and general population samples: Prevalence, correlates, and functions. Am J Orthopsychiatry 68, 609-620.

Bryan, C., Bryan, A. 2014. Nonsuicidal self-injury among a sample of United States Military Personnel and Veterans enrolled in college classes. J Clin Psychol. 1-12.

Bryan, C.J., Cukrowicz, K.C., West, C.L., et al., 2010. Combat experience and the acquired capability for suicide. J Clin Psychol., 66, 1044-1056.

Bryan, C.J., Hernandez, A.M., Allison, S., Clemans, T. 2013. Combat exposure and suicide risk in two samples of military personnel. J Clin Psychol. 69, 64-77. 
Bryan, C.J., Rudd, M.D., Wertenberger, E., Peterson, A.L., Young-McCaughan, S., Mintz, J. et al., 2015. Nonsuicidal self-injury as a prospective predictor of suicide attempts in a clinical sample of military personnel. Compr Psychiat. 59, 1-7.

Bullman, T.A., Kang, H.K., 1994. Posttraumatic stress disorder and the risk of traumatic deaths among Vietnam veterans. J Nerv Ment Dis. 182, 604-610.

Chapman, A.L., Gratz, K.L., Brown, M.Z., 2006. Solving the puzzle of deliberate self-harm: The experiential avoidance model. Behav Res Ther. 44, 371-394.

Crosby, A.E., Ortega, L., Melanson, C. 2011. Self-directed Violence Surveillance: Uniform Definitions and Recommended Data Elements. Retrieved from: http://www.cdc.gov/violenceprevention/pdf/Self-Directed-Violence-a.pdf.

DeBeer, B.B., Kimbrel, N.A., Meyer, E.C., Gulliver, S.B., Morissette, S.B. 2014. Combined posttraumatic stress (PTSD) and depressive symptoms interact with social support to predict suicidal ideation in Operation Enduring Freedom and Operation Iraqi Freedom (OEF/OIF) veterans. Psychiat Res. 216, 357-362.

First, M.B., Spitzer, R.L., Gibbon, M., Williams, J.B. 1994. Structural Clinical Interview for Axis I DSM-IV Disorders (Version 2nd). New York, NY: Biometrics Research Department.

Gerberding, J.L., Binder, S., 2003. Report to Congress on mild traumatic brain injury in the United States: Steps to prevent a serious public health problem. National Center for Inquiry Prevention and Control. Atlanta, GA: Centers for Disease Control and Prevention. 
Gratz, K.L., Tull, M.T., Levy, R., 2014. Randomized controlled trial and uncontrolled 9-month follow-up of an adjunctive emotion regulation group therapy for deliberate self-harm among women with borderline personality disorder. Psychol Med. 44, 2099-2112.

Hamza, C.A., Stewart, S.L., Willoughby, T., 2012. Examining the link between nonsuicidal selfinjury and suicidal behavior: A review of the literature and an integrated model. Clin Psychol Rev. 32, 482-495.

Joiner, T.E., 2005. Why people die by suicide. Harvard University Press: Cambridge.

Kang, H.K., Bullman, T.A., 2008. Risk of suicide among US veterans after returning from the Iraq or Afghanistan war zones. JAMA. 300, 652-653.

Kaplan, M.S., Huguet, N., McFarland, B.H., Newsom, J.T. 2007. Suicide among male veterans: A prospective population-based study. J Epidemiol Commun H. 61, 619-624.

Kessler, R.C., Borges, G., Walters, E.E. 1999. Prevalence of and risk factors for lifetime suicide attempts in the National Comorbidity Survey. Arch Gen Psychiat. 56, 617-626.

Kimbrel, N.A., Calhoun, P., Elbogen, E., Brancu, M., VA Mid-Atlantic MIRECC Registry Workgroup, Beckham, J.C. 2014b. The factor structure of psychiatric comorbidity among Iraq/Afghanistan-ear veterans and its relationship to violence, incarceration, suicide attempts, and suicidality. Psychiat Res. 220, 397-403.

Kimbrel, N.A., Evans, L.D., Patel, A.B., Wilson, L.C., Meyer, E.C., Gulliver, S.B., et al. 2014c. The Critical Warzone Experiences (CWE) Scale: Initial Psychometric properties and association with PTSD, anxiety, and depression. Psychiat Res. 220, 1118-1124.

Kimbrel, N.A., Johnson, M.E., Clancy, C., Hertzberg, M., Collie, C., Van Voorhees, E.E. et al., 2014a. Deliberate self-harm in male Iraq/Afghanistan veterans seeking treatment for posttraumatic stress disorder. J Trauma Stress. 27, 474-477. 
Kimbrel, N.A., DeBeer, B.B., Meyer, E.C., Silvia, P.J., Beckham, J.C., Young, K.A. et al. (2015). An examination of the broader effects of warzone experiences on returning Iraq/Afghanistan veterans' psychiatric health. Psychiat Res. 226, 78-83.

Kimbrel NA, Gratz KL, Tull MT, Morissette, S.B., Meyer, E.C., DeBeer, B.B. et al., 2015a. Non-suicidal self-injury as a predictor of active and passive suicidal ideation among Iraq/Afghanistan veterans. Psychiat Res. 227, 360-362.

Klonsky, E.D., 2011. Non-suicidal self-injury in United States adults: prevalence, sociodemographics, topography and functions. Psychol Med. 41, 1981-1986.

Klonsky, E.D., May, A.M., Glenn, C.R., 2013. The relationship between nonsuicidal self-injury and attempted suicide: Converging evidence from four samples. J Abnorm Psychol. 122, 231-237.

Linehan, M., 1993. Cognitive-behavioral treatment of borderline personality disorder. Guilford: New York.

Maguen, S., Metzler, T.J., Bosch, J., Marmar, C.R., Knight, S.J., Neylan, T.C. 2012. Killing in combat may be independently associated with suicidal ideation. Depress Anxiety. 29, 918-923.

Miller, D., 1994. Women Who Hurt Themselves. New York: Basic Books.

Morissette, S.B., Woodward, M., Kimbrel, N.A., Meyer E.C., Kruse, M.I., Dolan, S. et al., 2011. Deployment-related TBI, persistent post-concussive symptoms, PTSD, and depression in OEF/OIF Veterans. Rehabil Psychol. 56, 340 - 350.

Nock, M.K., Hwang, I., Sampson, N.A., Kessler, R.C. 2010. Mental disorders, comorbidity, and suicidal behavior: Results from the National Comorbidity Survey Replication. Mol Psychiatr. 15, 868-876. 
Nock, M.K., Joiner, T.E.Jr, Gordon K.H., Lloyd-Richardson, E., Pinstein, M.J. 2006. Nonsuicidal self-injury among adolescents: Diagnostic correlates and relation to suicide attempts. Psychiat Res. 144(1), 65-72.

Nock, M.K., Kessler, R.C. 2006. Prevalence of and risk factors for suicide attempts versus suicide gestures: Analysis of the National Comorbidity Survey. J Abnorm Psychol. 115, 616-623.

Posner, K., Brown, G.K., Stanley, B., Brent, D.A., Yershova, K.V., Oquendo, M.A. et al., 2011. The Columbia-Suicide Severity Rating Scale: Initial validity and internal consistency findings from three multisite studies with adolescents and adults. Am J Psychiat. 168(12), 1266-1277.

Remafedi, G., French, S., Story, M., Resnick, M.D., Blum, R. 1998. The relationship between suicide risk and sexual orientation: Results of a population-based study. Am J Public Health. 88, 57-60.

Sacks, M., Flood, A., Dennis, M.F., Hertzberg, M.A., Beckham, J.C. 2008. Self-mutilative behaviors in male veterans with posttraumatic stress disorder. J Psychiat Res. 42, 487494.

Selby, E.A., Anestis, M.D., Bender, T.W., et al., 2010. Overcoming the fear of lethal injury: Evaluating suicidal behavior in the military through the lens of the InterpersonalPsychological Theory of Suicide. Clin Psychol Rev. 30, 298-307.

Silverman, M.M., Berman, A.L., Sanddal, N.D., O’Carroll, P.W., Joiner, T.E. 2007. Rebuilding the Tower of Babel: A revised nomenclature for the study of suicide and suicidal behaviors part 1: Background, rationale, and methodology. Suicide Life Threat. 27, 248263. 
Suyemoto, K.L., 1998. The functions of self-mutilation. Clin Psychol Rev. 18, 531-554.

U.S. Department of Veterans Affairs, 2013. Mental health services, suicide prevention program. Suicide Data Report. Retrieved from: http://www.va.gov/opa/docs/Suicide-Data-Report2012-final.pdf.

Vasterling, J.J., 2008. TBI screening interview [Unpublished measure]. Boston, MA: VA Boston Healthcare System. 


\section{Figure Caption}

Figure 1. Association between Lifetime History of Nonsuicidal Self-Injury (NSSI) and Lifetime History of Suicide Attempts among Iraq/Afghanistan-era Veterans. Chi-square tests revealed that lifetime history of NSSI was positively associated with history of 1 or more suicide attempts, $\chi^{2}(1)=53.779, p<0.001$, and history of multiple suicide attempts, $\chi^{2}(1)=34.398, p<0.001$, at the bivariate level. 


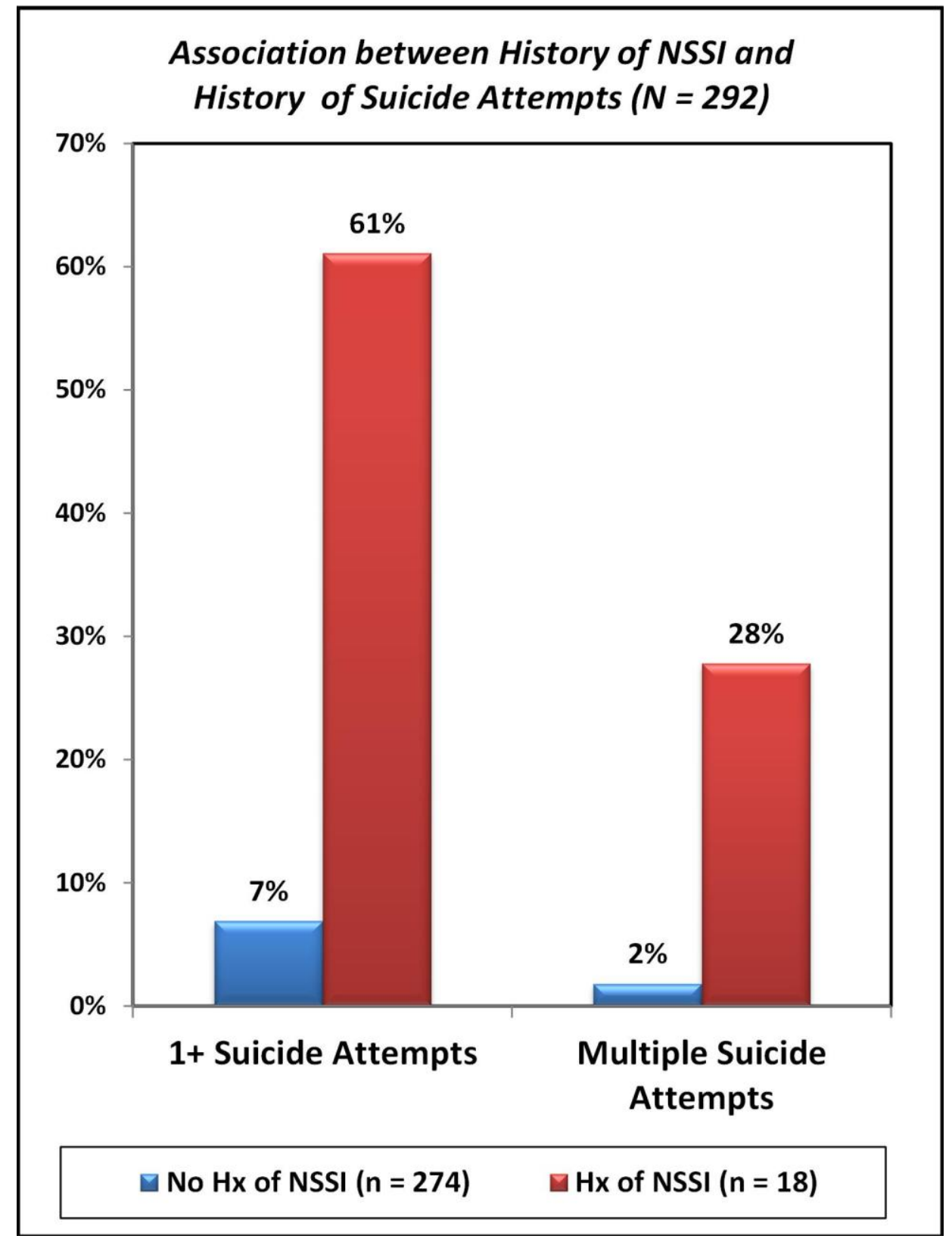

Figure 1 
Table 1

Summary of Bivariate Associations Between Suicide Attempts and Predictors

\begin{tabular}{|c|c|c|c|}
\hline \multicolumn{4}{|c|}{ Bivariate Associations Between Suicide Attempts and Categorical Predictors } \\
\hline$\underline{\text { Variable Name }}$ & $\underline{\text { Lifetime Attempts }}$ & $\underline{\text { Test Statistic }}$ & $p$-value \\
\hline \multicolumn{4}{|l|}{ Gender } \\
\hline Female & $16.8 \%$ & $\chi^{2}(1)=6.590$ & 0.01 \\
\hline Male & $7.1 \%$ & & \\
\hline \multicolumn{4}{|l|}{ Race } \\
\hline Non-White & $10.2 \%$ & $\chi^{2}(1)=0.000$ & 0.98 \\
\hline White & $10.2 \%$ & & \\
\hline \multicolumn{4}{|l|}{ Sexual Orientation } \\
\hline Heterosexual & $9.8 \%$ & $\chi^{2}(1)=1.064$ & 0.30 \\
\hline Non-Heterosexual & $17.6 \%$ & & \\
\hline \multicolumn{4}{|l|}{ TBI } \\
\hline No History of TBI & $11.0 \%$ & $\chi^{2}(1)=0.066$ & 0.78 \\
\hline History of TBI & $10.0 \%$ & & \\
\hline \multicolumn{4}{|l|}{ PTSD } \\
\hline No History of PTSD & $7.6 \%$ & $\chi^{2}(1)=2.379$ & 0.12 \\
\hline History of PTSD & $13.1 \%$ & & \\
\hline \multicolumn{4}{|l|}{ Depression } \\
\hline No History of Depression & $3.8 \%$ & $\chi^{2}(1)=16.001$ & $<0.0001$ \\
\hline History of Depression & $18.0 \%$ & & \\
\hline \multicolumn{4}{|l|}{ Alcohol Dependence } \\
\hline No History of Alcohol Dependence & $7.0 \%$ & $\chi^{2}(1)=7.660$ & 0.006 \\
\hline History of Alcohol Dependence & $17.6 \%$ & & \\
\hline \multicolumn{4}{|l|}{ Nonsuicidal Self-Injury } \\
\hline No History of Nonsuicidal Self-Injury & $6.9 \%$ & $\chi^{2}(1)=53.779$ & $<0.0001$ \\
\hline History of Nonsuicidal Self-Injury & $61.1 \%$ & & \\
\hline
\end{tabular}

\begin{tabular}{lccc}
\hline \multicolumn{4}{c}{ Bivariate Association Between Suicide Attempts and Years of Age } \\
\hline Mean (SD) & $\frac{\text { Test Statistic }}{\mathrm{t}=3.790}$ & $\frac{p \text {-value }}{<0.0001}$ \\
No History of Suicide Attempts & $32.4(7.6)$ & & \\
History of Suicide Attempts & $\frac{\text { Mean (SD) }}{5.1(5.8)}$ & $\frac{\text { Test Statistic }}{\mathrm{t}=-2.585}$ & $\frac{p \text {-value }}{0.01}$ \\
\hline Bivariate Association Between Suicide Attempts and Combat Exposure \\
\hline $\begin{array}{l}\text { No History of Suicide Attempts } \\
\text { History of Suicide Attempts }\end{array}$ & $8.1(7.8)$ & & \\
\hline
\end{tabular}


Table 2

Summary of Logistic Regression Models Predicting Suicide Attempts among Veterans

\begin{tabular}{|c|c|c|c|}
\hline & Nagelkerke $R^{2}$ & $\begin{array}{l}\text { Odds } \\
\text { Ratio }\end{array}$ & $\begin{array}{c}\text { 95\% Confidence } \\
\text { Intervals }\end{array}$ \\
\hline Step 1 & 0.211 & & \\
\hline Gender & & $0.224 * *$ & $0.082-0.609$ \\
\hline Age & & $0.910 * *$ & $0.862-0.962$ \\
\hline Race & & 0.968 & $0.401-2.388$ \\
\hline Sexual Orientation & & 1.475 & $0.354-6.153$ \\
\hline Combat Exposure & & $1.996 * *$ & $1.238-3.220$ \\
\hline Step 2 & 0.274 & & \\
\hline Gender & & $0.240 *$ & $0.076-0.754$ \\
\hline Age & & $0.919 * *$ & $0.869-0.972$ \\
\hline Race & & 0.873 & $0.346-2.203$ \\
\hline Sexual Orientation & & 1.122 & $0.251-5.020$ \\
\hline Combat Exposure & & $1.907 *$ & $1.076-3.379$ \\
\hline Traumatic Brain Injury & & 1.122 & $0.401-3.143$ \\
\hline PTSD & & 0.514 & $0.178-1.481$ \\
\hline Depression & & $3.150 *$ & $1.101-9.010$ \\
\hline Alcohol Dependence & & 2.186 & $0.868-5.502$ \\
\hline Step 3 & 0.429 & & \\
\hline Gender & & $0.131 * *$ & $0.033-0.524$ \\
\hline Age & & $0.926^{* *}$ & $0.873-0.981$ \\
\hline Race & & 0.833 & $0.304-2.287$ \\
\hline Sexual Orientation & & 0.849 & $0.122-5.910$ \\
\hline Combat Exposure & & $3.204^{* *}$ & $1.541-6.662$ \\
\hline Traumatic Brain Injury & & 0.751 & $0.242-2.329$ \\
\hline PTSD & & 0.364 & $0.109-1.217$ \\
\hline Depression & & 2.150 & $0.696-6.636$ \\
\hline Alcohol Dependence & & 1.961 & $0.696-5.523$ \\
\hline Nonsuicidal Self-Injury & & $28.494 * * *$ & $6.903-117.614$ \\
\hline
\end{tabular}

Note PTSD = posttraumatic stress disorder. Gender was coded as $0=$ female, $1=$ male. Race categories were collapsed across groups in order to increase statistical power and coded as $0=$ non-white, $1=$ white. Sexual orientation categories were collapsed across groups in order to increase statistical power and coded as $0=$ heterosexual, $1=$ non-heterosexual. The PTSD, depression, alcohol dependence, TBI, and nonsuicidal self-injury variables were all lifetime history variables. $* p<$ $0.05 ; * * p<0.01 ; p<0.001$ 